\title{
EFEITO RESIDUAL DE CHLORIMURON-ETHYL APLICADO EM MISTURA COM GLYPHOSATE NA DESSECAÇÃO DE PLANTAS DANINHAS
}

\author{
Tarcísio de O. Valente $^{1}$ e Maurício A. Cavazzana ${ }^{2}$ \\ ! Engenheiro Agrônomo, M.Sc., Professor. UFMS/CEUD/DCA. Rod. Dourados-Ithaum, km 12. Caixa Postal 533. Dourados, MS 79840-070. \\ tvalente@ceud.ufms.br \\ ${ }^{2}$ Acadêmico de Agronomia. UNESP/Ilha Solteira. Rua Carlos Gomes, 11. Araçatuba, SP 16100-310
}

\begin{abstract}
RESUMO
O trabalho teve por objetivo avaliar o efeito residual de chlorimuron-ethyl no controle da planta daninha Bidens pilosa, proveniente de sementes germinadas após a dessecação, em semeadura direta de soja, em um Latossolo Vermelho-Amarelo, fase Cerrado. Foram aplicados os seguintes tratamentos: glyphosate $(720 \mathrm{~g} / \mathrm{ha})$, glyphosate (1440 g/ha), glyphosate+chlorimuronethyl $(720+10 \mathrm{~g} / \mathrm{ha})$, glyphosate + chlorimuron-ethyl $(720+20 \mathrm{~g} / \mathrm{ha})$, glyphosate+chlorimuron-ethyl + flumioxazin $(720+20+40 \mathrm{~g} /$ ha), glyphosate+2,4-D $(720+460 \mathrm{~g} / \mathrm{ha})$ e uma testemunha sem capina. As aplicações foram feitas com pulverizador costal, com pressão constante mantida por $\mathrm{CO}_{2}$ a $2,1 \mathrm{~kg} / \mathrm{cm}^{2}$, com barra de dois metros, portando bicos tipo leque 110:02 e uma vazão de 200 l/ha. Aos 15, 30 e 45 dias após a aplicação foram avaliados o número de plantas e a percentagem de controle de Bidens pilosa. Os resultados mostraram um controle excelente de B. pilosa a partir dos 15 dias após a aplicação. Houve uma redução na população de B. pilosa, chegando a zero nos tratamentos de glyphosate+chlorimuron-ethyl $(720+20 \mathrm{~g} / \mathrm{ha}) \mathrm{ou}$ de glyphosate+chlorimuron-ethyl+flumioxazin $(720+20+40 \mathrm{~g} / \mathrm{ha})$, indicando boas perspectivas do uso de chlorimuron-ethyl em mistura com glyphosate durante a dessecação.
\end{abstract}

Palavras-chave: herbicida, plantio direto, flumioxazin, 2,4-D.

\section{ABSTRACT \\ Residual effect of chlorimuron-ethyl tank mixed with glyphosate applied as a burndown treatment for pre-site preparation in soybean}

An experiment was conducted on a Cerrado sandy soil during 1999/2000. The objective of the experiment was to evaluate the residual effect of chlorimuron-ethyl tank mixed with glyphosate applied as burndown treatment for pre-site preparation, to control Bidens pilosa in preemergence. The experimental design was a randomized complete block with four replications. Treatments were as follows: glyphosate at 720 and $1440 \mathrm{~g} / \mathrm{ha}$, glyphosate+chlorimuron-ethyl at $720+10$ and $720+20 \mathrm{~g} / \mathrm{ha}$, glyphosate+chlorimuron-ethyl+flumioxazin at $720+20+40 \mathrm{~g} /$ ha and glyphosate $+2,4-\mathrm{D}$ at $720+460 \mathrm{~g} / \mathrm{ha}$. An untreated check was also included. Treatments were applied by a $\mathrm{CO}_{2}$ backpack sprayer, using $2.1 \mathrm{~kg} / \mathrm{cm}^{2}$ of pressure, with four $110.02 \mathrm{XR}$ nozzles, and spray volume of $200 \mathrm{l} / \mathrm{h}$. Evaluations of percentage control and number of Bidens pilosa per square meter were made at 15 , 30 and 45 days after treatments. Chlorimuron-ethyl + glyphosate at $20+720 \mathrm{~g} /$ ha and chlorimuron-ethyl + flumioxazin + glyphosate at $20+40+720 \mathrm{~g} /$ ha showed excellent residual control for Bidens pilosa. Consequently, chlorimuron-ethyl can be used as an alternative and safe herbicide in soybean for pre site preparation tank mixed with glyphosate.

Key words: weed, herbicides, no till management, flumioxazin, 2,4-D. 


\section{INTRODUÇÃO}

No cultivo da soja, que constitui na principal atividade agrícola da região do Cerrado, as plantas daninhas têm sido motivo de preocupação permanente entre os agricultores, pois tem causado redução drástica no rendimento de grãos. Um dos fatores responsáveis pela queda de produção é a interferência das plantas daninhas à cultura da soja, podendo causar redução de até $42 \%$ (Blanco et al., 1978). O período crítico de competição da maioria das plantas daninhas com a soja situase entre 30 e 45 dias após a emergência da cultura (Blanco et al., 1978). Para cada acréscimo de um grama na produção de matéria seca de planta daninha, há uma redução de 2,67 a 23,48 $\mathrm{kg} /$ ha na produtividade da cultura (Velini, 1997). Para dificultar o surgimento de biotipos resistentes e realizar o controle destas plantas infestantes, há necessidade de se usar mistura de dois ou mais princípios ativos diferentes, além de praticar a rotação de culturas (Sociedade Brasileira da Ciência das Plantas Daninhas, 2000).

A utilização de misturas de herbicidas tem crescido muito na condução das lavouras, principalmente na dessecação da cobertura vegetal para semeadura direta da soja. A utilização de herbicidas residuais em misturas com dessecantes permite uma redução direta nos custos de aplicação, com a redução do número de aplicações. Esta prática permite também, menor exposição do aplicador, menos compactação dos solos e possivelmente, melhor controle das plantas daninhas no início de seu estabelecimento. A eficiência dos herbicidas residuais em mistura com os dessecantes depende basicamente do teor de matéria orgânica e da capacidade de troca catiônica (CTC) dos solos e da precipitação pluviométrica ocorrida após a aplicação. Quanto maior o teor de matéria orgânica e a CTC, maior será a adsorção dos herbicidas e menor a disponibilidade dos produtos, com menor eficiência de controle. A precipitação pluviométrica contribui para a lixiviação dos produtos da palha para a superfície do solo, dispondo-os em contato com as sementes das plantas daninhas.

Rodrigues et al. (2000), utilizando solo pesado e com boa palhada na superfície, verificou que o controle de ervas foi inferior a $30 \%$ quando usados residuais com dessecantes antes da semeadura, enquanto que na modalidade seqüencial os índices sempre foram superior a 85\%, em relação á aplicação convencional. O mesmo autor, avaliando o comportamento de herbicidas pré-emergentes aplicados sobre a cobertura morta em plantio direto, verificou que todos os herbicidas utilizados foram interceptados pela cobertura morta, antes da irrigação de $20 \mathrm{~mm}$ feita 24 horas após a aplicação e que os produtos apresentaram índices de lixiviação variando de 5 a $90 \%$. Os resultados mostraram também que os herbicidas mais lixiviáveis ficaram mais retidos na palha quando em mistura com os menos lixiviáveis. Siqueira et al. (2000) estudou a influencia dos resíduos de chlorimuron-ethyl na cultura da soja sobre as características agronômicas do milheto em sucessão e verificou efeito visual de fitotoxicidade em milheto de $40 \%$ aos 21 e 28 DAS (dias após a semeadura) e de $20 \%$ aos 35 e 42 DAS.

Este trabalho teve como objetivo avaliar a eficiência agronômica do efeito residual de chlorimuron-ethyl quando utilizado no manejo de dessecação, em mistura com glyphosate, nas condições do Cerrado.

\section{MATERIAL E MÉTODOS}

O ensaio foi conduzido em Latossolo Vermelho-Amarelo, fase Cerrado na Fazenda SM2, da Sementes Maggi, município de Rondonópolis, MT, durante a safra 1999/2000. Utilizou-se a cultura da soja, variedade Uirapuru, plantada em $02 /$ $12 / 99$, utilizando espaçamento de $0,45 \mathrm{~m}$, densidade média de 16 sementes por metro linear e adubada com $390 \mathrm{~kg} / \mathrm{ha}$ do formulado 04-20-20, em sistema de plantio direto. A análise física do solo apresentou as seguintes características: areia $49,9 \%$, argila $43,4 \%$, silte $6,7 \%$. A análise química apresentou $\mathrm{pH}$ (água) 5,8, matéria orgânica $29 \mathrm{~g} / \mathrm{dm}^{3} \mathrm{e}$ CTC de $8,3 \mathrm{cmol} /$ c $\mathrm{dm}^{3}$. Os tratos culturais, à exceção do controle de plantas daninhas, que se constituiu nos tratamentos estudados, seguiram as recomendações técnicas para a região (EMBRAPA, 1995). Os tratamentos estão especificados na Tabela 1 e foram aplicados uma única vez, em pós-emergência, para a dessecação, no dia 02/12/99, exceto o glyphosate+2,4-D, que foi aplicado sete dias antes da semeadura. Utilizou-se o delineamento experimental em blocos casualizados com quatro repetições, onde os tratamentos foram aplicados em parcelas com $24 \mathrm{~m}^{2}$ de área total $(3 \mathrm{~m} \times 8 \mathrm{~m})$ e $16 \mathrm{~m}^{2}$ de área aplicada $(2 \mathrm{~m}$ x $8 \mathrm{~m}$ ). A planta daninha alvo na dessecação e no controle foi picão-preto (Bidens pilosa) que se encontrava com uma densidade de 122 indivíduos por metro quadrado. A aplicação por aspersão foi realizada com um pulverizador manual pressurizado a gás carbônico, mantido à pressão constante de $2,1 \mathrm{~kg} / \mathrm{cm}^{2}$, contendo uma barra com quatro bicos espaçados de $0,5 \mathrm{~m}$, portando pontas de jato plano XR 110.02 e proporcionando um volume de calda de $200 \mathrm{l} / \mathrm{ha}$. As plantas de picão-preto, no momento da aplicação apresentavam-se adultas, com maturidade definida, já com as sementes liberadas ao solo.

As aplicações foram feitas em condições favoráveis ao bom desempenho dos produtos, ou seja, a aplicação do 2,4-D no dia $24 / 11 / 99$, com temperatura de $27^{\circ} \mathrm{C}$ e umidade relativa de $78 \%$ e os outros tratamentos no dia $01 / 12 / 99$, com temperatura de $28^{\circ} \mathrm{C}$ e $79 \%$ de umidade relativa.

As variáveis avaliadas foram a percentagem de dessecação, a fitotoxicidade aos 7, 15 e 30 DAA (dias após a aplicação), seguindo a escala de conceitos, que varia de 0 a 100 , sendo 0 (zero) nenhum efeito e 100 (cem) morte total da 
Tabela 1. Tratamentos estudados e algumas características dos herbicidas. Rondonópolis-MT, 1999/2000.

\begin{tabular}{lccc}
\hline \multicolumn{1}{c}{ Nome comum } & $\begin{array}{c}\text { Dose } \\
(\mathbf{g} / \mathbf{h a})\end{array}$ & Nome comercial & Concentração \\
\hline Glyphosate & 720 & Roundup & $360 \mathrm{~g} / \mathrm{l}$ \\
Glyphosate & 1440 & Roundup & $360 \mathrm{~g} / \mathrm{l}$ \\
Glyphosate+chlorimuron ethyl & $720+10$ & Gliphogan+Classic & $360 \mathrm{~g} / \mathrm{l}+250 \mathrm{~g} / \mathrm{kg}$ \\
Glyphosate+chlorimuron ethyl & $720+20$ & Gliphogan+Classic & $360 \mathrm{~g} / \mathrm{l}+250 \mathrm{~g} / \mathrm{kg}$ \\
$\begin{array}{l}\text { Glyphosate+chlorimuron- } \\
\text { ethyl+flumioxazin }\end{array}$ & $720+20+40$ & Gliphogan+Classic+Flumizin & $360 \mathrm{~g} / \mathrm{l}+250 \mathrm{~g} / \mathrm{kg}+500 \mathrm{~g} / \mathrm{kg}$ \\
$\begin{array}{l}\text { Glyphosate+2,4 D } \\
\text { Testemunha }\end{array}$ & $720+460$ & Roundup+U46 & $360 \mathrm{~g} / 1+460 \mathrm{~g} / \mathrm{l}$ \\
\hline
\end{tabular}

planta (Frans \& Talbert, 1977). O efeito residual no controle de Bidens pilosa, foi avaliado medindo-se o número de plantas emergidas por metro quadrado aos 15,30 e 45 DAA. Foi avaliada também a altura das plantas de soja no final do ciclo da cultura. As médias de fitotoxicidade foram interpretadas seguindo os conceitos da escala de avaliação do Australian Weeds Committee (1979). Os dados de altura de plantas e o número de plantas emergidas após a dessecação foram submetidos à análise de variância e teste de média.

\section{RESULTADOS E DISCUSSÃO}

As plantas de soja não apresentaram, aparentemente, efeitos fitotóxicos visíveis quando se utilizou chlorimuronethyl ou 2,4-D em mistura com glyphosate na dessecação. As avaliações realizadas aos 14,21 e 42 DAA (dias após as apli- cações) revelaram $0 \%$ de fitotoxicidade nas três datas, para todos os tratamentos. $\mathrm{O}$ crescimento da planta foi influenciado significativamente pelo tratamento glyphosate $+2,4-\mathrm{D}$, aplicado sete dias antes da semeadura (Tabela 2). Este efeito poderá ser explicado pela baixa CTC apresentada por este solo, além do elevado teor de areia e baixo teor de matéria orgânica, tornando o 2,4-D disponível para a planta. Pode-se inferir também que a elevada precipitação ocorrida durante a germinação das sementes da soja pode ter contribuído para a disponibilidade do herbicida à cultura, afetando assim o seu desenvolvimento (Tabela 2). A dessecação foi bem sucedida, com controle muito bom, como pode ser observado na Tabela 3 e na Figura 1 (a) e (b).

A eficiência do efeito residual de chlorimuron-ethyl aplicado em mistura com glyphosate foi excelente a partir de $10 \mathrm{~g} /$ ha (Tabela 4), provocando redução do número de plantas emergidas de Bidens pilosa (Figura 2). As misturas chlorimuron-ethyl $+2,4-D(720+20 \mathrm{~g} / \mathrm{ha})$ e glyphosate $+2,4-\mathrm{D}$ apresentaram também controle eficiente (Figura 2).

Tabela 2. Resultados médios da altura das plantas de soja, aos 45 dias após a dessecação. Rondonópolis-MT, $1999 / 2000$.

\begin{tabular}{lcc}
\hline \multicolumn{1}{c}{ Tratamento } & $\begin{array}{c}\text { Dose } \\
\text { (g/ha) }\end{array}$ & $\begin{array}{c}\text { Altura } \\
\text { (cm) }\end{array}$ \\
\hline Glyphosate & 720 & $43,3 \mathrm{a} \mathrm{b}$ \\
Glyphosate & 1440 & $45,1 \mathrm{a}$ \\
Glyphosate+chlorimuron-ethyl & $720+10$ & $49,7 \mathrm{a}$ \\
Glyphosate+chlorimuron-ethyl & $720+20$ & $49,2 \mathrm{a}$ \\
Glyphosate+chlorimuron-ethyl+flumioxazin & $720+20+40$ & $48,1 \mathrm{a}$ \\
Glyphosate+2,4-D & $720+460$ & $41,4 \mathrm{~b}$ \\
Testemunha & - & $36,6 \mathrm{~b}$ \\
\hline F (1\%) & & 6,94 \\
\hline C.V. $(\%)$ & & 8,7 \\
\hline
\end{tabular}

Médias seguidas de mesma letra, na coluna, não diferem entre si estatisticamente ao nível de $5 \%$ de probabilidade pelo teste de $\mathrm{F}$. 
Tabela 3. Resultados médios da avaliação visual do controle de Bidens pilosa estabelecida (estádio $80 \%$ de sementes maduras) durante a dessecação. Rondonópolis-MT, 1999/2000.

\begin{tabular}{lcccc}
\hline \multirow{2}{*}{\multicolumn{1}{c}{ Tratamento }} & Dose & \multicolumn{3}{c}{ Controle (\%) } \\
\cline { 3 - 5 } & $(\mathrm{g} / \mathbf{h a})$ & 7 DAT & 15 DAT & 30 DAT \\
\hline Glyphosate & 720 & 78 & 97 & 100 \\
Glyphosate & 1440 & 89 & 100 & 100 \\
Glyphosate+chlorimuron-ethyl & $720+10$ & 85 & 100 & 100 \\
Glyphosate+chlorimuron-ethyl & $720+20$ & 85 & 100 & 100 \\
Glyphosate+chlorimuron-ethyl+flumioxazin & $720+20+40$ & 84 & 100 & 100 \\
Glyphosate+2,4-D & $720+460$ & 100 & 100 & 100 \\
Testemunha & - & 0 & 0 & 0 \\
\hline F (1\%) & & & & \\
\hline C.V. $(\%)$ & &
\end{tabular}

Tabela 4. Resultados médios da avaliação do número de plantas $/ \mathrm{m}^{2}$ de Bidens pilosa germinada aos 15,30 e 45 dias após a dessecação. Rondonópolis-MT, 1999/2000.

\begin{tabular}{lcccc}
\hline \multirow{2}{*}{\multicolumn{1}{c}{ Tratamento }} & \multirow{2}{*}{$\begin{array}{c}\text { Dose } \\
\text { (g/ha) }\end{array}$} & 15 DAT & 30 DAT & 45 DAT \\
\cline { 3 - 5 } & 720 & $499 \mathrm{~b}$ & $288 \mathrm{~b}$ & $158 \mathrm{~b}^{1}$ \\
Glyphosate & 1440 & $371 \mathrm{~b}$ & $391 \mathrm{~b}$ & $153 \mathrm{~b}$ \\
Glyphosate & $720+10$ & $59 \mathrm{a}$ & $37 \mathrm{a}$ & $3 \mathrm{a}$ \\
Glyphosate+chlorimuron-ethyl & $720+20$ & $22 \mathrm{a}$ & $25 \mathrm{a}$ & $0 \mathrm{a}$ \\
Glyphosate+chlorimuron-ethyl & $520+20+40$ & $5 \mathrm{a}$ & $13 \mathrm{a}$ & $0 \mathrm{a}$ \\
Glyphosate+chlorimuron-ethyl+flumioxazin & $720+460$ & $118 \mathrm{a}$ & $69 \mathrm{a}$ & $35 \mathrm{a}$ \\
Glyphosate+2,4-D & - & $138 \mathrm{a}$ & $118 \mathrm{a}$ & $34 \mathrm{a}$ \\
Testemunha & & $10,39^{* *}$ & $11,56 * *$ & $7,60^{* *}$ \\
\hline F (1\%) & & 68,0 & 64,4 & 93,3 \\
\hline C.V. $(\%)$ & & &
\end{tabular}

${ }^{1}$ Médias seguidas de mesma letra, na coluna, não diferem entre si estatisticamente ao nível de $5 \%$ de probabilidade pelo teste $\mathrm{F}$.
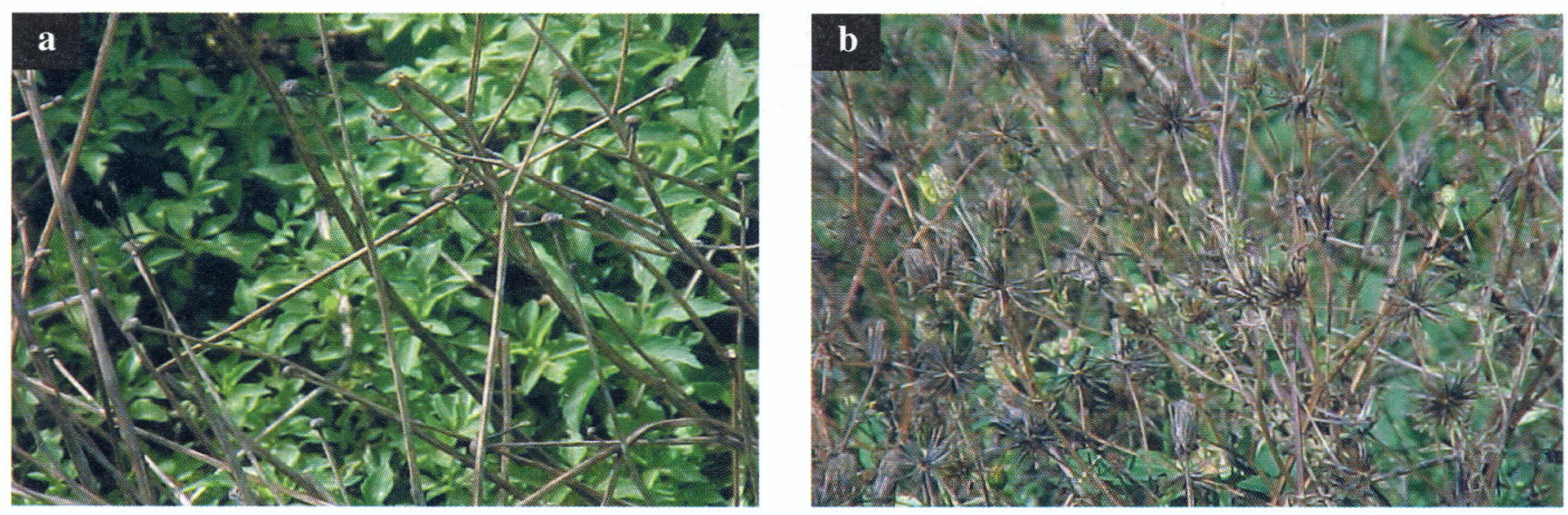

Figura 1. Aspecto geral da sementeira (a) e da dessecação (b) de Bidens pilosa após a aplicação dos produtos. 

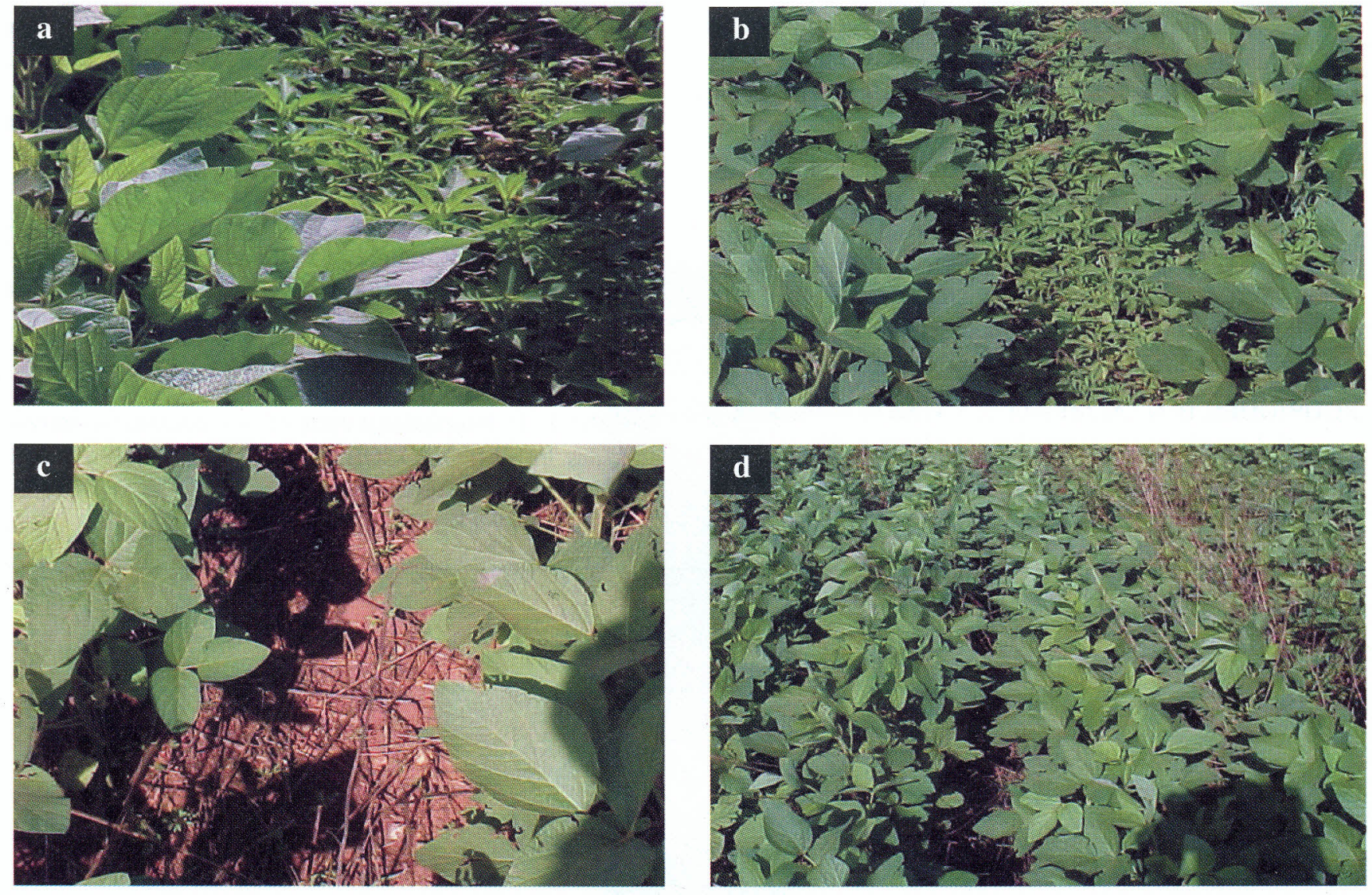

Figura 2. Efeito residual dos tratamentos glyphosate (720 g/ha) (a), glyphosate $(1440 \mathrm{~g} / \mathrm{ha})(\mathrm{b})$, glyphosate+chlorimuronethyl+flumioxazin $(720+20+40 \mathrm{~g} / \mathrm{ha})(\mathrm{c})$, glyphosate+chlorimuron-ethyl $(720+40 \mathrm{~g} / \mathrm{ha})(\mathrm{d})$, aplicados durante a dessecação de Bidens pilosa para semeadura direta da soja.

\section{CONCLUSÕES}

O herbicida chlorimuron-ethyl (Classic) apresentou efeito residual para o controle da sementeira de Bidens pilosa na cultura da soja, em solo de Cerrado. A mistura de chlorimuron-ethyl a $10 \mathrm{~g} / \mathrm{ha}$ com glyphosate (Gliphogan) a $720 \mathrm{~g} / \mathrm{ha}$, utilizada na dessecação da vegetação para o plantio da cultura da soja, controlou Bidens pilosa. A recomendação da aplicação de chlorimuron-ethyl em mistura com glyphosate para dessecação de Bidens pilosa visando o controle residual desta espécie na soja, em região de Cerrados, poderá ser feita seguindo as práticas culturais da região.

\section{LITERATURA CITADA}

AUSTRALIAN WEEDS COMMITTEE. Guidelines for field evaluation of herbicides. Canberra Australian Government Publishing Service, 1979, p. A1-5.

BLANCO, H. G., OLIVEIRA, D., ARAUJO, J. B. M. Período crítico de competição de uma comunidade natural de mato em soja (Glycine max (L.) Merril.). Biológico v.44, n.11, p. 299-305, 1978.

EMBRAPA. Centro de Pesquisa Agropecuária do Oeste. Soja: recomendações técnicas para Mato Grosso do Sul e Mato Grosso. Dourados: EMBRAPA-CPAO, 1995. $121 \mathrm{p}$. (EMBRAPA-CPAO. Circular Técnica, 1). 
FRANS, R. E.; TALBERT, R. E. Design of field experiments and the measurement and analysis of plant responses. In: TRUELOVE, B. Ed. Research Methods in Weed Science. 2. Ed., C. 2, 1977.

RODRIGUES, N. B.; FORNAROLLI, D. A.; LIMA, J. de; CAETANO, E. S.; MORAES, V. J. Comportamento de herbicidas residuais aplicados em mistura com dessecantes em plantio direto. In: CONGRESSO BRASILEIRO DA CIÊNCIA DAS PLANTAS DANINHAS, 22, 2000, Foz do Iguaçu. Resumos... Londrina: Sociedade Brasileira da Ciência das Plantas Daninhas, 2000. p. 379.

RODRIGUES, B. N.; ALMEIDA, F. S. Guia de herbicidas. 4. ed. Londrina, Edição dos Autores, 1998. 648p.

SIQUEIRA, J. L. de; SILVA, J. B. da; SOUZA, F. I. de; PEIXOTO, M. F. Efeito de herbicidas aplicados na cultura da soja sobre as características agronômicas do milheto em sucessão. In: CONGRESSO BRASILEIRO DA CIÊNCIA DAS PLANTAS DANINHAS, 22, 2000, Foz do Iguaçu. Resumos... Londrina: Sociedade Brasileira da Ciência das Plantas Daninhas, 2000. p. 526.

SOCIEDADE BRASILEIRA DA CIÊNCIA DAS PLANTAS DANINHAS. Comitê Brasileiro de Resistência de Plantas aos Herbicidas. Identificação e manejo de plantas daninhas resistentes aos herbicidas. Londrina: SBCPD, 2000.32p.

VELINI, E. D. Interferência entre plantas daninhas e cultivadas. In: SIMPÓSIO SOBRE HERBICIDAS E PLANTAS DANINHAS. Resumos... Dourados: EMBRAPA-CPAO, 1997.p. 29-49. 